\title{
The advantages of the use of discs containing single agents in disc diffusion testing of the susceptibility of Aeromonas salmonicida to potentiated sulphonamides
}

\author{
Iain Douglas ${ }^{a}$, Neil M. Ruane ${ }^{\mathrm{b}}$, Michelle Geary ${ }^{\mathrm{b}}$, Cyril Carroll $^{\mathrm{a}}$, \\ Gerard T.A. Fleming ${ }^{a}$, Joanne McMurray ${ }^{a}$, Pete Smith ${ }^{a}$ \\ ${ }^{a}$ Department of Microbiology, National University of Ireland Galway, Galway, Ireland \\ ${ }^{\mathrm{b}}$ Fish Health Unit, Marine Institute, Oranmore, Galway, Ireland
}

\begin{abstract}
The susceptibilities of 106 strains of Aeromonas salmonicida to trimethoprim/sulfamethoxazole (SFT) were determined in two laboratories using the Clinical and Laboratory Standards Institute's M42-A disc diffusion protocols. The data generated by the use of discs containing $25 \mu \mathrm{g}$ SFT (SFT 25 ) allowed the strains to be placed into two groups. Strains in one group (17 strains) generated no inhibition zones and the zones obtained from the other 89 strains were distributed over a wide range but showed no natural division into separate sub-classes. A further investigation performed by one of the participating laboratories, of the susceptibility of 91 of these 106 strains used discs containing $100 \mu \mathrm{g}$ sulfmethoxazole $\left(\mathrm{SFM}_{100}\right)$ and $5 \mu \mathrm{g}$ trimethoprim $\left(\mathrm{TMP}_{5}\right)$. Application of normalised resistance interpretation to these data allowed the estimation of epidemiological cut-off values for WT strains of $\geq 9 \mathrm{~mm}_{\text {for }} \mathrm{SFM}_{100}$ and $\geq 21 \mathrm{~mm}$ for TMP . This investigation demonstrated the presence of three distinct phenotypic classes, one containing strains manifesting wild type susceptibility to both agents, another containing strains manifesting non-wild type susceptibility to both and a third containing strains manifesting wild type susceptibility with respect to TMP but non-wild type with respect to SFM. Analysis demonstrated the inability of $\mathrm{SFT}_{25}$ discs to generate data that allowed the separate identification of strains that were fully susceptible to both TMP and SFM from those that were fully susceptible to TMP but were not fully susceptible to SFM.

It is recommended that, in investigation of the susceptibility to potentiated sulphonamides of isolates from diseased fish, separate discs, containing the individual components of the mixture, should be employed.
\end{abstract}

1. Introduction

There is a long history of the use of potentiated sulphonamide agents in the control of bacterial diseases of fish (Aoki et al., 1972). In the US and Canada the most commonly used products are those containing a mixture of ormetoprim and sulfadimethoxine in a ratio of 1:19 (SFO). In Europe the preferred mixture has been trimethoprim and sulfamethoxazole in the same ratio (SFT). Currently there are Marketing Authorisations for the use in aquaculture 
of 6 products containing trimethoprim/ sulfmethoxazole in nine European countries (Guichard and Licek, 2006).

In the early 1970s Aoki et al. (1972) published on the plasmid location of genes involved in resistance to sulphonamides and plasmids encoding resistance to sulphonamides and to both sulphonamides and trimethoprim have been detected in isolates made in Scotland and Ireland (Hedges et al., 1985; Inglis et al., 1993). The very significant advances in our understanding of the genetics of resistance have recently been documented by Sørum (2006). However, despite the long period during which these mixtures have been in use and our extensive knowledge of the molecular biology of the genetic mechanisms encoding resistance to them, there are still no standard validated breakpoints for assessing the clinical significance of in-vitro measures of susceptibility to SFT and SFO of isolates made from fish disease outbreaks.

In a recent survey (Smith, 2006, 2007) of current practice, 29 of 31 responding laboratories reported using disc diffusion methods to determine the susceptibility of clinical isolates. Twenty-two $(75 \%)$ of the laboratories reported the breakpoint values they employed to provide a clinical interpretation of inhibition zones generated by discs containing SFT. There was, however, considerable variation in the breakpoint values employed and those being used to define resistance by the 18 laboratories using $25 \mu \mathrm{g}$ discs ranged from $8 \mathrm{~mm}$ to $23 \mathrm{~mm}$.

Standard methods for performing in-vitro disc diffusion susceptibility tests (M42-A) (CLSI, 2006a) have recently been issued by the Clinical Laboratory and Standards Institute (CLSI). This CLSI protocols is functionally equivalent to that previously published by Alderman and Smith (2001) but is associated with acceptable ranges for control strains. Miller and Reimschuessel (2006) have recently used the MIC and disc diffusion data generated by the application of the CLSI protocols (2006a,b) to 217 strains of Aeromonas salmonicida to estimate appropriate epidemiological cut-off values (EUCAST, 2000; Kahlmeter et al., 2003; CLSI, 2002) for SFO.

In this work the M42-A protocols (CLSI, 2006a) were used to provide data on the susceptibility of 106 strains of A. salmonicida to SFT. Given the predominance of disc diffusion methods in laboratories dealing with clinical isolates (Smith, 2006, 2007), it was decided to confine the study to data generated by this method and to use the data from two independent laboratories to provide the necessary internal controls.
2. Materials and methods

\subsection{Bacteria}

\subsubsection{Inter-laboratory studies with $25 \mu \mathrm{g}$ trimethoprim/ sulfmethoxazole discs}

One hundred and six strains of $A$. salmonicida were used in this work. All strains had been isolated from diseased fish, were brown pigmented and reacted with antiserum (BioNor MONO AQUA). These strains were received from Scotland (33strains), Norway (30strains), Denmark(17strains), France (15 strains) and Ireland (11 strains). The control strain $A$. salmonicida NCIMB 1102 (equivalent to ACTC 33658) was obtained from the National Collection of Industrial and Marine Bacteria (UK).

\subsubsection{Studies in Laboratory 2 with $100 \mu \mathrm{g}$ sulfmethoxazole discs and $5 \mu \mathrm{g}$ trimethoprim discs}

Ninety-one of the 106 strains of A. salmonicida were used in this portion of the work. This strain set included 33 from Scotland, 27 from Norway, 14 from Denmark, 14 from France and 3 from Ireland.

\subsection{Media}

Bacteria were routinely cultured using a Mueller Hinton Agar or Mueller Hinton Broth purchased from Mast Group (Bootle, UK). Incubations were performed at $22 \pm 2{ }^{\circ} \mathrm{C}$ for 44 $-48 \mathrm{~h}$.

\subsection{Discs}

Discs containing $25 \mu \mathrm{g}$ trimethoprim/sulfmethoxazole (1:19)(SFT 25$), 100 \mu \mathrm{g}$ sulfmethoxazole $\left(\mathrm{SFM}_{100}\right)$ and $5 \mu \mathrm{g}$ trimeth-oprim $\left(\mathrm{TMP}_{5}\right)$ were obtained from Mast Group (Bootle, UK).

\subsection{Diffusion assay}

All disc diffusion assays were performed according to the protocols specified in M42-A (CLSI, 2006a). Suspensions were made in saline and incubations were at $22 \pm 2{ }^{\circ} \mathrm{C}$ and zones were read after $44-48 \mathrm{~h}$. A. salmonicida NCIMB 1102 was used as the control strain.

\subsection{Statistical analysis}

Normalised resistance interpretation (NRI) was performed according to the method of Joneberg et al. (2003), Kronvall (2003) and Kronvall et al. (2003). Peak values were established using 5 point rolling means and the mean and standard deviation of the real distribution of fully susceptible (wild type) strains were calculated using a plot of probit values against zonesize. In each case 6 probit values were plotted and these included the first value $\mathrm{N} 5$ and the next five values lower than this. Epidemiological cut-off values were set at two and a half standard deviations below the mean (Smith et al., in press). 


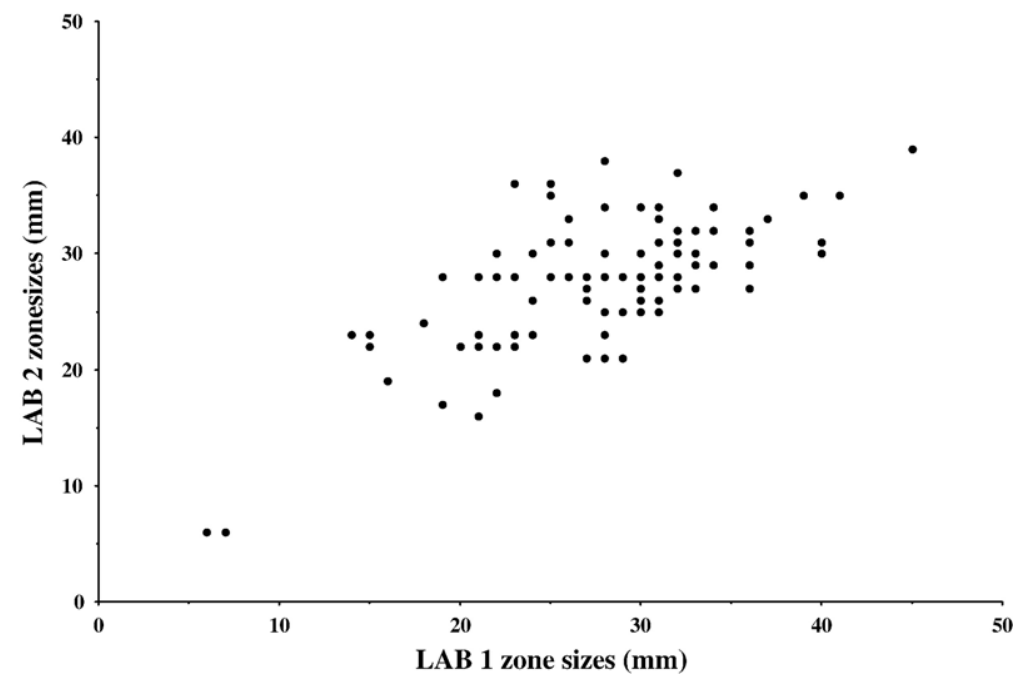

Fig.1.Scatter-plot of thezonesizes recorded by the twolaboratoriesusing $25 \mu \mathrm{g}$ trimethoprim/sulfamethoxazole(1:19) discs againstall 106strains of $A$. salmonicida. The vertical line indicates the cut-off value estimated by NRI analysis.

All other statistical analyses were performed using InStat 3.0 (GraphPad Inc).

\section{Results}

\subsection{Studies with $S F T_{25}$ discs}

During these experiments the zone sizes for $\mathrm{SFT}_{25}$ discs against the control strain NCIMB1102 were determined nine times by each laboratory and all determinations lay within the acceptable range $(24-39 \mathrm{~mm}$ ) specified by M42-A (CLSI, 2006a) for tests performed under the conditions used in this work. The mean of the determinations by Laboratory 1 and 2 were $26 \pm 1.7 \mathrm{~mm}$ and $28 \pm 0.8 \mathrm{~mm}$ respectively.

With respect to the zone sizes they obtained for the 106 strains tested both laboratories obtained similar distributions (Fig. 1) and no gross discrepancies were observed. It should be noted that the individual points in Fig. 1 might represent more

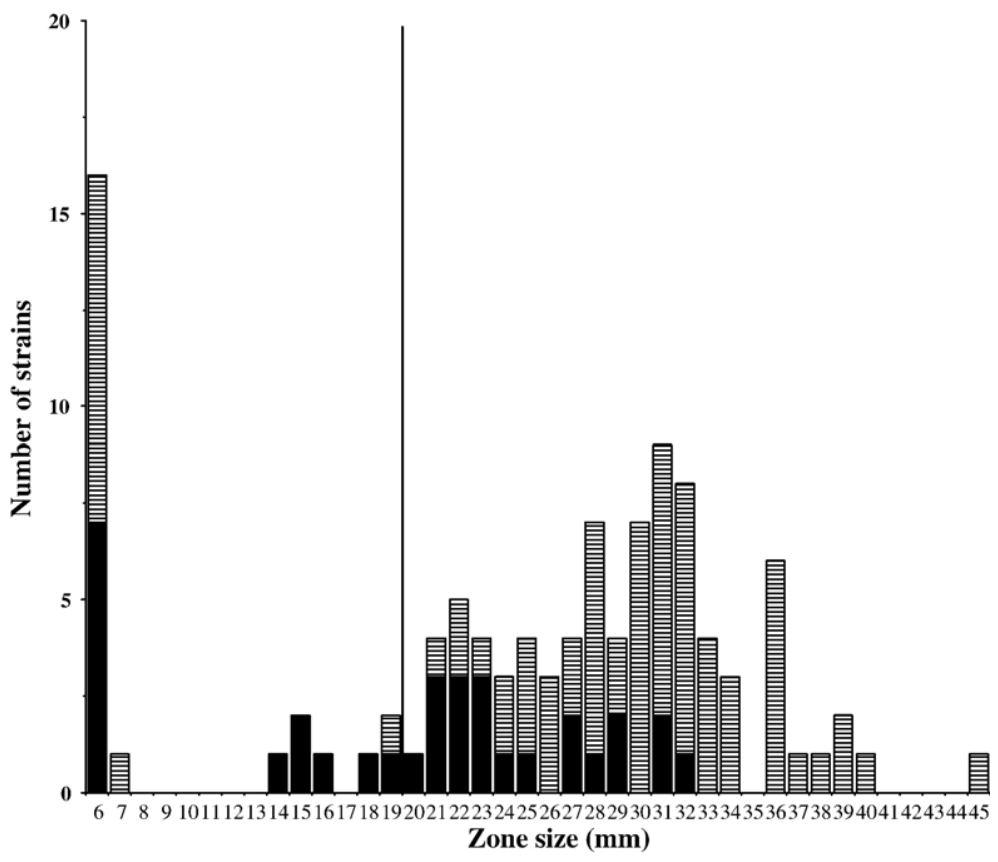

Fig. 2. Inhibition zone diameters recorded by Laboratory 1 using $25 \mu \mathrm{g}$ trimethoprim/sulfamethoxazole (1:19) discs against all $106 \mathrm{strains}$ of A. salmonicida. Zones generated by Scottish strains are shown as solid bars. Zones for isolates from other countries are shown as striped bars. 
Table1

Zone size data recorded using $25 \mu \mathrm{g}$ trimethoprim/sulfamethoxazole $(1: 19)$ discs

\begin{tabular}{lccccccc}
\hline & \multicolumn{2}{l}{$\begin{array}{l}\text { All countries } \\
(n=89)\end{array}$} & \multicolumn{2}{l}{$\begin{array}{l}\text { All countries } \\
\text { except Scotland } \\
(n=63)\end{array}$} & & \multicolumn{2}{l}{$\begin{array}{l}\text { Scotland } \\
(n=26)\end{array}$} \\
\cline { 2 - 3 } \cline { 7 - 8 } & LAB 1 & LAB 2 & LAB 1 & LAB 2 & & LAB 1 & LAB 2 \\
\hline Mean & 28.2 & 28.0 & 30.7 & 29.7 & & 23.0 & 24.3 \\
SD & 5.9 & 4.7 & 5.1 & 3.8 & & 5.1 & 4.9 \\
\% CV & 21 & 17 & 17 & 13 & & 22 & 20 \\
Median & 29 & 28 & 31 & 30 & & 22.5 & 23 \\
Min & 14 & 16 & 19 & 21 & & 14 & 16 \\
Max & 41 & 38 & 45 & 39 & 32 & 36 \\
\hline
\end{tabular}

Statistical parameters of the data obtained from the 89 strains of A. salmonicida that gave zones $\mathrm{N} 7 \mathrm{~mm}$, analysed by country of isolation.

than one strain. Visual examination of the $\mathrm{SFT}_{25}$ data from the two laboratories (Figs. 2 and 5) suggested that the zone sizes fell into two clusters. Both laboratories recorded zones $\leq 7 \mathrm{~mm}$ for the same 17 strains and the zone sizes they recorded for the other 89 strains fell into a diffuse grouping that could not, on visual examination, be divided into discrete sub-groups.

\subsubsection{Frequency of strains with zones $\leq 7 \mathrm{~mm}$ with $S F T_{25}$ discs}

With respect to $\mathrm{SFT}_{25}$ discs, zones of inhibition of $\leq 7 \mathrm{~mm}$ were recorded by both laboratories for the same 17 strains and these could, therefore, be classified as non-wild type (NWT) with respect to SFT (SFT $\mathrm{NWT}_{\mathrm{NT}}$ ). Application of Fisher's exact test indicated that, in none of the national strain sets, were the frequencies of $\mathrm{SFT}_{\mathrm{NWT}}$ strains significantly different from that observed in the 106-strain set.

\subsubsection{Analysis of strains yielding zones $\mathrm{N} 7 \mathrm{~mm}$ with $\mathrm{SFT}_{25}$ discs}

Table 1 presents the statistical parameters of the $\mathrm{SFT}_{25}$ zone size distributions obtained by the two laboratories for the strains that generated zone sizes $\mathrm{N} 7 \mathrm{~mm}$. When the zones from all 89 strains were considered as one set (the 'All countries' columns in Table1), the central tendency of the data obtained by Laboratory $1 \& 2$ were remarkably similar (mean $28.2 \mathrm{~mm}$ and $28.0 \mathrm{~mm}$ respectively). However these laboratories recorded high ( $21 \%$ and $17 \%$ respectively) coefficients of variation for these data. Despite these high degrees of variance, a visual examination of the distribution of the data for these strains (Figs. 2 and 5) provided no grounds for proposing the existence of discrete sub-groups within the 89 strains. There were, however, two lines of evidence that suggested that such sub-groups were in fact present.

The first line of evidence derived from NRI analysis. Using this method to estimate the cut-off values for the wild type (WT) group resulted in values of $\geq 20 \mathrm{~mm}$ for Laboratory 1 and $\geq 19 \mathrm{~mm}$ for Laboratory 2. However, for Laboratory 1 , seven strains ( $8 \%$ ) gave zones that were $\mathrm{N} 7 \mathrm{~mm}$ but b2 $2 \mathrm{~mm}$. For Laboratory 2, three strains (3\%) gave zones N7 mm but b $19 \mathrm{~mm}$. Setting the cut-off value for WT strains at the NRI mean minus two and a half standard deviations has the result that this lower limit should encompass N99\% of all sensitive strains. Thus, the frequency with which both laboratories

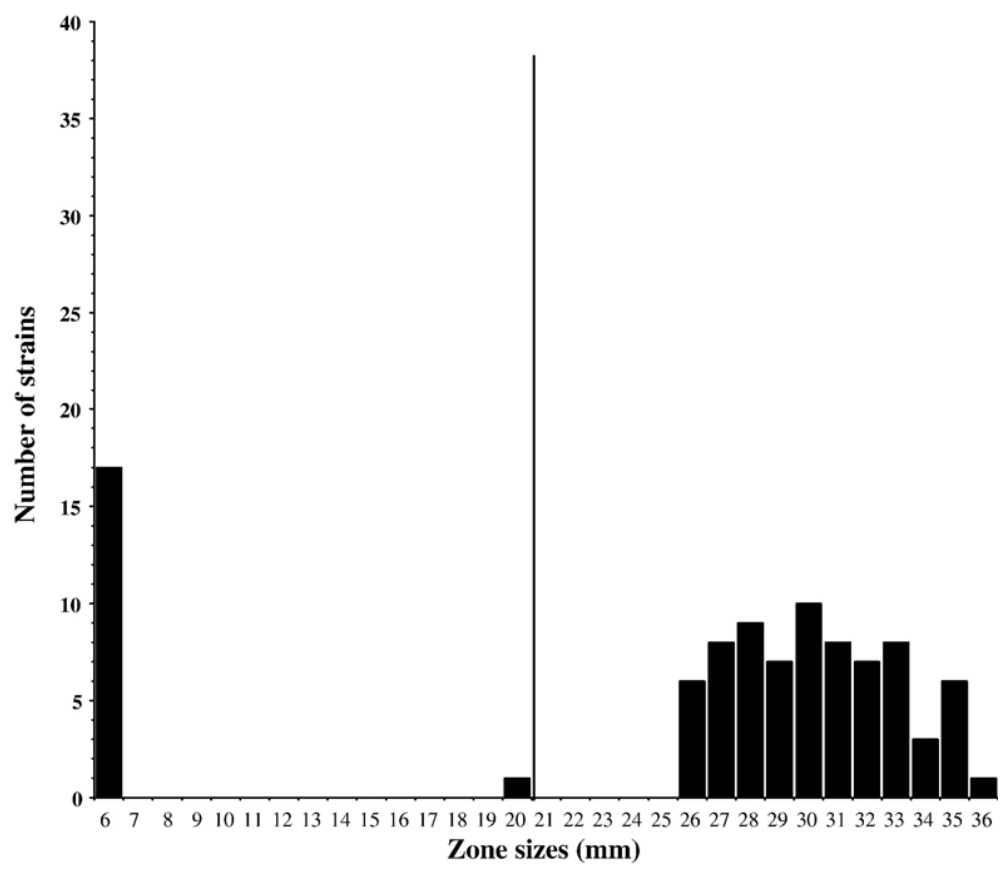

Fig. 3. Inhibition zone diameters recorded by Laboratory 2 using $5 \mu \mathrm{g}$ trimethoprim discs against 91 strains of $A$. salmonicida. 


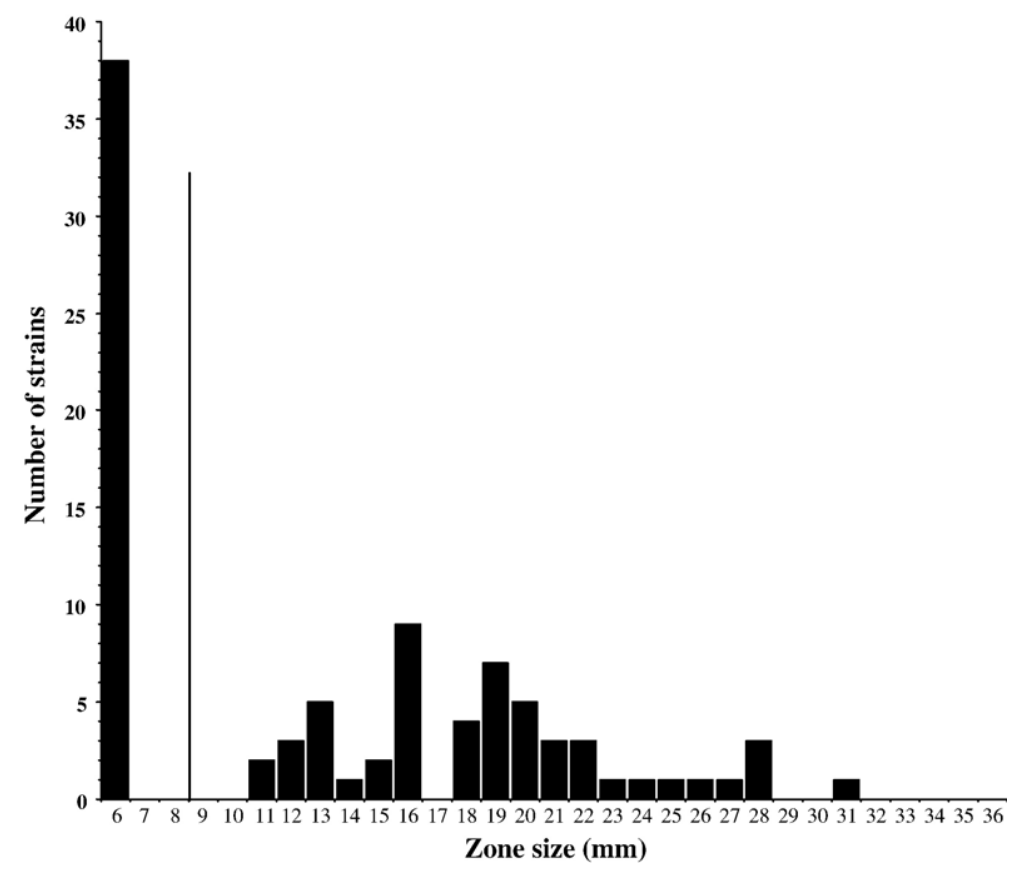

Fig. 4. Inhibition zone diameters recorded by Laboratory 2 using $100 \mu \mathrm{g}$ sulfamethoxazole discs against 91 strains of $A$. salmonicida.

recorded $\mathrm{SFT}_{25}$ zones below their cut-off value for WT strains but greater than $7 \mathrm{~mm}$, must be taken as evidence that a subpopulation manifesting reduced susceptibility was present in the 89 strain set.
The second line of evidence emerged when the 89 strain set data were analysed on the basis of the country of origin of the strains. Within the 89 strains that gave $\mathrm{SFT}_{25}$ zones N7 mm, twenty-six originated from Scotland. Table 1 presents the

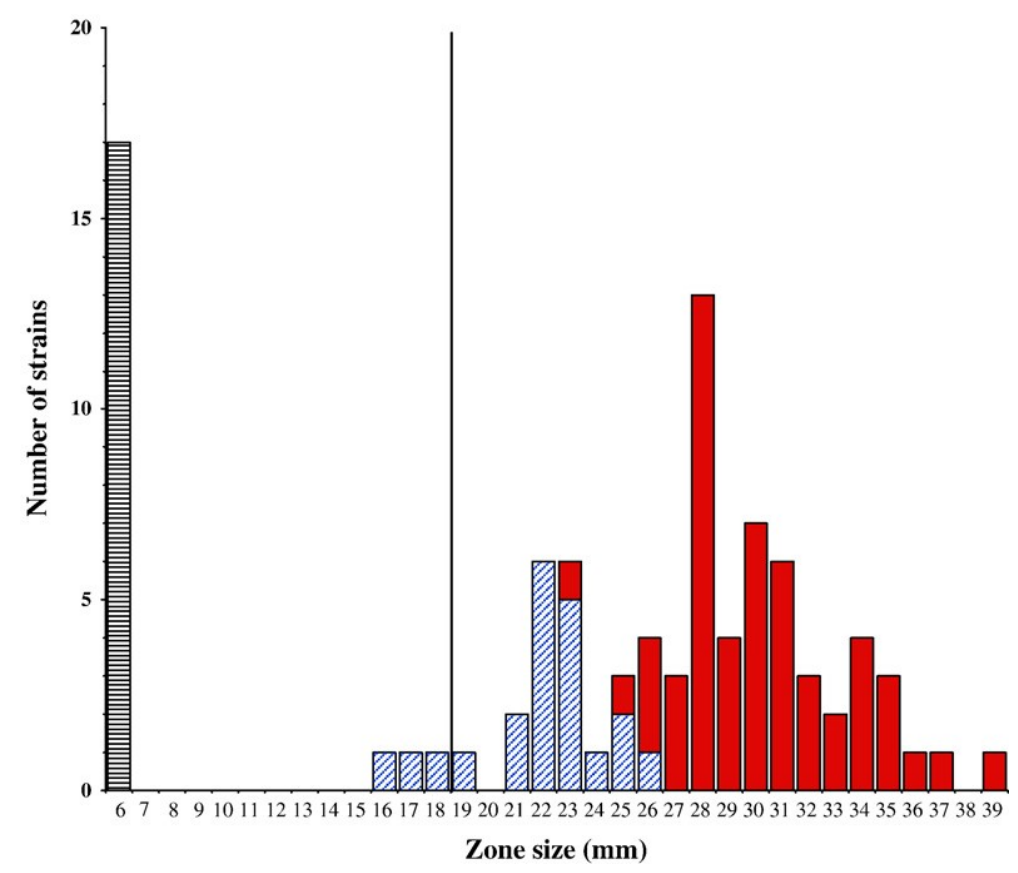

Fig. 5. Inhibition zone diameters recorded by Laboratory 2 using $25 \mu \mathrm{g}$ trimethoprim/sulfamethoxazole(1:19) discs against 91 strains of $A$. salmonicida. Solid bars indicate strains with a SFM $\mathrm{WT}_{\mathrm{WT}} / \mathrm{TMP}_{\mathrm{WT}}$ phenotype. Diagonally striped bars indicate strains withSFM $\mathrm{NWT}_{\mathrm{NT}} / \mathrm{TMP}_{\mathrm{WT}}$ phenotype and horizontally striped bars indicate those with a $\mathrm{SFM}_{\mathrm{NWT}} / \mathrm{TMP}_{\mathrm{NWT}}$ phenotype. The vertical line indicates the cut-off value estimated by NRI analysis. 
statistical parameters of the distributions of the 26 strains isolated in Scotland and the 63 strains isolated in the other countries. These statistical parameters demonstrate that, on average, the Scottish strains (means $23.0 \mathrm{~mm}$ and $24.3 \mathrm{~mm}$ ) were less susceptible than the others (means $30.7 \mathrm{~mm}$ and $29.7 \mathrm{~mm}$ ). Mann-Whitney analysis demonstrated that for both laboratories, the difference between the Scottish and nonScottish data sets were significant ( $p$ b0.001). The most plausible explanation of these significant differences was that the Scottish strain set contained some strains of reduced susceptibility. The high coefficients of variation recorded by both laboratories for the Scottish strains, however, suggest that not all the Scottish strains could be considered as manifesting reduced susceptibility. Fig. 2 separately identifies the data generated by Laboratory 1 from the 33 Scottish strains (solid bars) and from the 73 strains isolated in other countries (striped bars). This histogram demonstrates that, with respect to the 89 strains that gave zones $\mathrm{N} 7 \mathrm{~mm}$, some but not all of the Scottish strains gave zones that lay to the smaller end of the distribution. A similar analysis (not shown) of the data from Laboratory 2 gave essentially thesame results.

Taken together the NRI analysis and the analysis by country of origin provided strong evidence that the 89 strains that gave zones $\mathrm{N} 7 \mathrm{~mm}$ should not be treated as a diffuse but essentially homogeneous group. Rather these analyses suggest that there were at least two sub-populations within this group and that these sub-populations could not be identified using data from $\mathrm{SFT}_{25}$ discs.

\subsection{Studies with $S F M_{100}$ and $T M P_{5}$ discs}

In order to further investigate the susceptibility of strains that gave zones $\mathrm{N} 7 \mathrm{~mm}$ with $\mathrm{SFT}_{25}$ discs, an investigation was made of the zones generated by these discs and by discs containing $25 \mu \mathrm{g} \mathrm{SFT} 25,5 \mu \mathrm{g}$ trimethoprim $\left(\mathrm{TMP}_{5}\right)$ and $100 \mu \mathrm{g}$ sulfmethoxazole $\left(\mathrm{SFM}_{100}\right)$. This study included 91 of the 106 strains used in the inter-laboratory studies with $\mathrm{SFT}_{25}$ discs.

Figs. 3 and 4 present the distributions of zone sizes obtained for $\mathrm{TMP}_{5}$ and $\mathrm{SFM}_{100}$ discs respectively. In each case the distributions were essentially bi-modal. Application of NRI analysis led to an estimated cut-off value for WT strains of $\geq 21 \mathrm{~mm}$ for $\mathrm{TMP}_{5}$. This allowed 73 strains to be classified as WT, 17 as NWT with one strain being given an intermediate classification. With respect to the $\mathrm{SFM}_{100}$ data the cut-off value for WT strains was calculated to be $\geq 9 \mathrm{~mm}$. This limit led to the classification of 38 strains as NWT and 53 as WT. However, as there was considerable high zone end trailing in the $\mathrm{SFM}_{100}$ data, this limit should be treated with some caution. These classifications with respect to SFM can be compared with those produced for oxytetracycline (OTC) for the same strains (Smith et al., in press). Of strains classified as $\mathrm{SFM}_{\mathrm{NWT}} 98 \%$ were also classified as OTC $\mathrm{NWT}_{\mathrm{N}}$ but of those classified as SFM WT $_{\text {only }} 19 \%$ were classified as OTC $\mathrm{NWT}_{\mathrm{NWT}}$.

Using these $\mathrm{SFM}_{100}$ and $\mathrm{TMP}_{5}$ cut-off values all 91 strains could be classified as belonging to one of three groups. One group contained 53 strains classified as WT with respect to
SFM and WT or intermediate with respect to TMP (SFMwT/ $\left.\mathrm{TMP}_{\mathrm{WT}}\right)$. The second group contained 21 strains, 17 of which originated in Scotland, that were classified as NWT with respect to SFM but WT with respect to TMP (SFM $\mathrm{SWT}_{\mathrm{NW}}$ $\left.\mathrm{TMP}_{\mathrm{WT}}\right)$. The third group of 17 strains were those classified as NWT with respect to both agents $\left(\mathrm{SFM}_{\mathrm{NWT}} / \mathrm{TMP}_{\mathrm{NWT}}\right)$. No strains were observed to be SFM $\mathrm{WT}_{\mathrm{WT}} / \mathrm{TMP}_{\mathrm{NWT}}$.

Fig. 5 presents the zone sizes recorded for the 91 strains using $\mathrm{SFT}_{25}$ discs, classified by their susceptibility to SFMand TMP. These data demonstrate that strains classified as $\mathrm{SFM}_{\mathrm{NWT}} / \mathrm{TMP} \mathrm{WT}_{\mathrm{W}}$ generated smaller zones for SFT discs than those classified as fully susceptible to both agents.

\section{Discussion}

\subsection{Analysis of $\mathrm{SFT}_{25}$ disc data}

Waterworth (1978) reported the World Health Organisation recommendation that, in using disc diffusion methods to test susceptibility to potentiated sulphonamides, the susceptibilities to the two components should be determined independently. Current practice in laboratories serving aquaculture appears not to follow this recommendation. In a recent survey (Smith, 2006) 22 of 23 European laboratories reported testing for susceptibility to SFT by disc diffusion. Of these, 18 reported using $\mathrm{SFT}_{25}$ discs and four reported using SFT discs with 10 -fold higher content. However, none of these laboratories reported using discs containing SFM or TMP. Recently published standard testing protocols appear to follow this practice. The disc diffusion protocols specified by both Alderman and Smith (2001) and M42-A (CLSI, 2006a), which are functionally identical, both recommend the use of $\mathrm{SFT}_{25}$ discs and neither make any recommendation with respect to discs containing SFM or TMP.

The data generated by Laboratory 2 from the

application of $\mathrm{SFM}_{100}$ and $\mathrm{TMP}_{5}$ discs demonstrated the existence of three, distinct phenotypic classes. One class was comprised of strains fully susceptible to both agents $\left(\mathrm{SFM}_{\mathrm{WT}} / \mathrm{TMP}_{\mathrm{WT}}\right)$, the second class was comprised of strains fully susceptible to neither $\left(\mathrm{SFM}_{\mathrm{NWT}} /\right.$ $\mathrm{TMP}_{\mathrm{NWT}}$ ) and a third that was comprised of strains not fully susceptible to SFM but fully susceptible to TMP $\left(\mathrm{SFM}_{\mathrm{NWT}} / \mathrm{TMP}_{\mathrm{WT}}\right)$. An important observation made in the inter-laboratory study was that data generated using $\mathrm{SFT}_{25}$ discs were unable to separate strains with these different susceptibilities to the individual components of SFT. A visual examination of the data generated from these discs allowed only a separation of these strains into two groups. One group was defined as those strains that gave zones of $7 \mathrm{~mm}$ and the other contained all the other89 strains. AlthoughNRIanalysis of theSFT discs 
data indicated that there were, in all probability, some strains of reduced susceptibility within this group of 89 strains, it wasnot capable of generating a limitor cut-off value that would have allowed theidentification of the two phenotypic classes that could be identified by the use of $\mathrm{SFM}_{100}$ and $\mathrm{TMP}_{5}$ discs. Fig. 5 demonstrates that the distributions of zone sizes generated by $\mathrm{SFT}_{25}$ discs for $\mathrm{SFM}_{\mathrm{NWT}} / \mathrm{TMP}_{\mathrm{WT}}$ strains overlapped with those obtained for strains classified as SFM ${ }_{\mathrm{WT}} / \mathrm{TMP}_{\mathrm{WT}}$. Given this degree of overlap it must be accepted that no form of analysis of data generated by the use of $\mathrm{SFT}_{25}$ disc data could ever provide a cut-off value that would allow the unambiguous differentiation of these two phenotypic classes.

The data generated in this work provide strong arguments in favour of the use of discs that contain the separate components of any potentiated sulphonamide combination. The data presented in Figs. 3 and 4 would suggest that NRI analysis would be capable of generating limit values for each component that could be used to detect the three phenotypic classes identified in this work.

\subsection{NRI cut-off values and clinical breakpoints}

Cut-off values for WT strains, calculated using NRI analysis, are not clinical breakpoints. These limit values are set using only laboratory data on susceptibility and can, therefore, relate only to in-vitro bacterial phenotypes. Clinical breakpoints, however, should relate to the prediction of the outcome of a therapy and can, therefore, be set only if consideration is also given to pharmacokinetic/pharmacodynamic (PK/PD) data and to data on clinical efficacy (Turnidge and Paterson, 2007). With respect to the therapy of disease in aquatic animals we are a long way from having the PK/PD and clinical efficacy data that would be required to set clinical breakpoints. In such situations, Kahlmeter et al. (2003) have argued that it is legitimate to use epidemiological cut-off values generated from distributions of MIC data, to estimate provisional and tentative breakpoints.

It is instructive to examine the breakpoints currently in use in European laboratories (Smith, 2006) in the light of the data presented here. In this work strains that were $\mathrm{SFM}_{\mathrm{NWT}} / \mathrm{TMP}_{\mathrm{WT}}$ gave zones in the range $15-26 \mathrm{~mm}$ for $\mathrm{SFT}_{25}$ discs. Of the 18 laboratories reporting breakpoints for $\mathrm{SFT}_{25}$ discs, 16 used limits of $\leq 14 \mathrm{~mm}$ to define resistance and one used a limit of $16 \mathrm{~mm}$. Thus, only one laboratory, which used a breakpoint of $23 \mathrm{~mm}$, would have regularly reported these $\mathrm{SFM}_{\mathrm{NWT}} / \mathrm{TMP}_{\mathrm{WT}}$ strains as resistant. Possibly more importantly, three- quarters of these laboratories used a breakpoint of $\leq 18 \mathrm{~mm}$ to define sensitivity and, therefore, presented with the data obtained in this work, would have classified and reported the majority of $\mathrm{SFM}_{\mathrm{NWT}} / \mathrm{TMP}_{\mathrm{WT}}$ strains as fully sensitive.

\subsection{Clinical significance of the $S F M_{N W T} / T M P_{W T}$ phenotype}

It is important to note that, at present, we do not have any data relating to the clinical significance of the levels of resistance to SFT manifest by strains that belong to the SFM $_{\text {NWT }} /$ TMP $_{\text {WT }}$ phenotypic class. Ringertz et al. (1997) have argued that, in interpreting susceptibility test data, emphasis should be placed on detecting resistance and that tentative breakpoints should, therefore, be set conservatively. There is an increasing demand that antimicrobial use in all fields, including aquaculture (WHO, 2006) should be prudent. In this context, it would be unwise to adopt interpretative criteria that would allow strains, which are not fully susceptible, to be classified as sensitive. In the absence of any evidence to the contrary, the demonstration of anything less than full susceptibility in a target bacterium should be treated as adequate grounds for issuing a recommendation that a proposed course of therapy should not be initiated.

\section{Conclusions}

The data presented in this work demonstrates that when $\mathrm{SFT}_{25}$ discs are used, the M42-A protocols (CLSI, 2006b) lack the sensitivity required to separate strains with different susceptibility phenotypes. They demonstrate, however that unambiguous separation of these phenotypic classes can be achieved if $\mathrm{SFM}_{100}$ and $\mathrm{TMP}_{5}$ discs are employed. This strongly argues that, in determining susceptibility to potentiated sulphonamides, best-practice guidelines should recommend the use of individual discs containing the separate components of the potentiated mixture, rather than those containing the mixture itself.

\section{Acknowledgements}

We acknowledge the support of our colleagues in providing the strains used in this work. French strains were supplied by the GDSAA (Groupement de Défense Sanitaire Aquacole d'Aquitaine, France) and the LD 40 (Laboratoire Départemental des Landes, France). The Norwegian strains were supplied by Henning Sørum (Department of Food Hygiene and Infectious Biology, Norwegian School of Veterinary Science, Oslo). The 
Scottish strains were supplied by David Bruno (The Marine Laboratory, Aberdeen). The Danish strains were supplied by Inger Dalsgaard (Danish Institute for Fisheries Research, Frederiksberg).

We also acknowledge the assistance of Göran Kronvall in performing the NRI analyses presented in this work.

\section{References}

Alderman, D.J., Smith, P, 2001. Development of draft protocols of standard reference methods for antimicrobial agent susceptibility testing of bacteria associated with fish diseases. Aquaculture 196, 211-243.

Aoki, T, Egusa, S., Yada, C., Watanabe, T , 1972. Studies of drug resistance and $\mathrm{R}$ factors in bacteria from pond-cultured salmonids. I. Amago (Oncorhynchus rhodurus macrostomus) and Yamame (Oncorhynchus masou ishikawae). Japanese Journal of Microbiology 16, 233-238.

CLSI, 2002. Development of in vitrosusceptibility testing criteria and quality control parameters for veterinary antimicrobial agents. Approved guideline M37-A2. Clinical and Laboratory Standards Institute, Wayne, Pennsylvania.

CLSI, 2006a. Methods for antimicrobial disk susceptibility testing of bacteriaisolated from aquatic animals. Approved guidelineM42-A. Clinical and Laboratory Standards Institute, Wayne, Pennsylvania.

CLSI, 2006b. Methods for broth dilution susceptibility testing of bacteria isolated from aquatic animals. Approved guideline M49-A. Clinical and Laboratory Standards Institute, Wayne, Pennsylvania.

EUCAST,2000. Terminology relating tomethodsfor thedetermination of susceptibility of bacteria to ant microbial agents. European Committee on Antimicrobial Susceptibility Testing Definitive documentE. Def 1.2. http://www.srga.org/Eucastwt/eucastdefinitions.htm2000May.

Guichard, B., Licek, E., 2006. A comparative study of antibiotics registered for use in farmed fish in European countries. Poster presented at the First OIE Global Conference on Aquatic Animal Health, October10, Bergen, Norway.

Hedges, R.W , Smith, P., Brazil, G., 1985. Resistance plasmids of Aeromonads. Journal of General Microbiology 131, 2091-2095.

Inglis, V., Yimer, E., Bacon, E.J., Ferguson, S., 1993. Plasmidmediated antibiotic resistance in Aeromonas salmonicida isolated from Atlantic salmon, Salmo salar L., in Scotland. Journal of Fish Diseases 16, 593-598.

Joneberg, J., Rylander, M., Galas, M.F., Carlos, C., Kronvall, G., 2003. Analysis of parameters and validation of method for normalized interpretation of antimicrobial resistance. International Journal of Antimicrobial Agents 21, 525-535.
Kahlmeter, G, Brown, D.F.J., Goldstein, F.W , MacGowan, A.P., Mouton, J.W., Österlund, A., Rodloff, A., Steinbakk, M., Urbaskova, P, Vatopoulos, A., 2003. European harmonization of MIC breakpoints for antimicrobial susceptibility testing of bacteria. Journal of Antimicrobial Chemotherapy 52, 145-148.

Kronvall, G., 2003. Determination of the real standard distribution of susceptible strains in zone histograms. International Journal of Antimicrobial Agents 22, 7-13.

Kronvall, G., Kahlmeter, G., Myhre, E., Galas, M.F., 2003. A new method for normalized interpretation of antimicrobial resistance from disk test results for comparative purposes. Clinical Microbiology and Infection 9, 120-132.

Miller, A , Reimschuessel, R., 2006. Epidemiological cutoff values for antimicrobial agents against Aeromonas salmonicida isolates determined by frequency distributions of minimal inhibitory concentration and diameter of zone of inhibition data. American Journal of Veterinary Research 67, 1837-1843.

Ringertz, S., Olsson-Liljequist, B., Kahlmeter, G., Kronvall, G., 1997. Antimicrobial susceptibility testing in Sweden; II. Species-related zone diameter breakpoints to avoid interpretive errors and guard against unrecognised evolution of resistance. Scandinavian Journal of Infectious Disease. Supplement 105, 8-12.

Smith, $\mathrm{P}, 2006$. Breakpoints for disc diffusion susceptibility testing of bacteria associated with fish diseases: a review of current practice. Aquaculture 261, 1113-1121.

Smith, P., 2007. A survey of methods and protocols currently being used to determine antimicrobial susceptibility of bacteria associated with fish disease. Bulletin of the European Association of Fish Pathologists 27, 18-22.

Smith, P., Ruane, N.M., Douglas, I., Carroll, C., Kronvall, G., Fleming, G.T.A., in press. Impact of inter-lab variation on the estimation of epidemiological cut-off values for disc diffusion susceptibility test data for Aeromonas salmonicida. Aquaculture.

Sørum, H., 2006. Antimicrobial drug resistance in fish pathogens. In: Aarestrup, F.M. (Ed.), Antimicrobial Resistance in Bacteria of Animal Origin. American Society for Microbiology Press, Washington, DC, USA, pp. 213-238.

Turnidge, J., Paterson, D.L., 2007. Setting and revising antibacterial susceptibility breakpoints. Clinical Microbiology Reviews 20, 391-408.

Waterworth, P.M., 1978. Quantitative methods for bacterial sensitivity testing. In: Reeves, D.S., Philips, I., Williams, J.D., Wise, R. (Eds.), Laboratory Methods in Antimicrobial Chemotherapy. Churchill Livingstone, Edinburgh, pp. 31-40.

WHO, 2006. Antimicrobial use in aquaculture and antimicrobial resistance. Report of a Joint FAO/OIE/WHO Expert Consultation on Antimicrobial Use in Aquaculture and Antimicrobial Resistance Seoul, Republic of Korea, 13 - 16 June. World Health Organization, Geneva. 